\title{
Bladder extragastrointestinal stromal tumor in an adolescent patient: A case-based review
}

\author{
FAN HE, ZHENQIANG FANG, PEI ZHU, WEI HUANG and LONGKUN LI \\ Department of Urology, Xinqiao Hospital, The Third Military Medical University, Chongqing 400037, P.R. China
}

Received April 11, 2014; Accepted July 2, 2014

DOI: $10.3892 / \mathrm{mco} .2014 .366$

\begin{abstract}
Extragastrointestinal stromal tumors (EGISTs) are mesenchymal tumors occurring outside the gastrointestinal tract, with histological, immunohistochemical and molecular genetic characteristics similar to those of gastrointestinal stromal tumors (GISTs). The immunohistochemical examination usually demonstrates a positive expression for CD117. GISTs are rare neoplasms and EGISTs are even less common. This is the report of a case of EGIST in the urinary bladder of a 15-year-old adolescent female patient who presented with painless gross hematuria. Pelvic computed tomography revealed an irregular soft tissue density mass, sized $5.7 \times 4.8 \mathrm{~cm}$, in the bladder. Partial cystectomy was performed in April, 2011. There was no recurrence during follow-up over the next 35 months, as determined by transabdoninal ultrasonography and cystoscopic examination. The patient in this study did not receive any molecular-targeted drugs. To the best of our knowledge, this is the first reported case of an EGIST of the urinary bladder in an adolescent patient.
\end{abstract}

\section{Introduction}

The majority of the mesenchymal tumors of the gastrointestinal tract are currently referred to as gastrointestinal stromal tumors (GISTs). The immunohistochemical examination commonly reveals a positive expression for CD117. Extragastrointestinal stromal tumors (EGISTs) are mesenchymal tumors occurring outside the gastrointestinal tract, with histological, immunohistochemical and molecular genetic characteristics very similar to those of GISTs (1). EGISTs are a rare clinical entity and, to date, there has been no report of bladder EGIST in non-adult patients. In this report, we present a mesenchymal tumor removed from the bladder of a 15-year-old female. The patient remains alive and disease-free 35 months after the surgery.

Correspondence to: Dr Longkun Li, Department of Urology, Xinqiao Hospital, The Third Military Medical University, 183 Xinqiaozheng Rd, Chongqing 400037, P.R. China

E-mail: 785517193@qq.com

Key words: extragastrointestinal stromal tumor, CD117, bladder, adolescent

\section{Case study}

A 15-year-old adolescent female patient was hospitalized following 2 weeks of painless gross hematuria with clots. On admission, the patient had stable vital signs, a pale appearance and no positive abdominal signs. On routine blood examination, the concentration of hemoglobin was $69 \mathrm{~g} / \mathrm{l}$. A B-mode ultrasound image indicated a urinary bladder neoplasm and pelvic computed tomography (CT) revealed an irregular soft tissue density mass, sized $5.7 \times 4.8 \mathrm{~cm}$, in the bladder, which exhibited uneven density and obscure boundaries (Fig. 1). The patient was subjected to partial cystectomy and the intraoperative exploration identified a sessile tumor, sized $5.7 \times 4.8 \times 3 \mathrm{~cm}$, in the upper wall of the bladder, which grew along the muscular layer and broke through the mucous layer with associated bleeding. There was no macroscopic invasion of the ureteral opening. The remaining bladder wall was smooth and exhibited no signs of neoplasia. On postoperative pathological examination, the tumor was composed of fusiform cells with abundant acidophilic cytoplasm and clavate nuclei, with both ends round and mostly blunt. The cells were mainly arranged in interwoven bundles or swirls and were mildly atypical (Fig. 2). The mitotic count was 5 per 50 high-power fields (HPFs). Immunohistochemically, the tumor cells strongly expressed CD117 (Fig. 3), vimentin and smooth muscle actin, but were negative for CD34, desmin and S-100 protein. The tumor was diagnosed as an EGIST. The patient in this study did not receive any molecular-targeted drugs due to financial difficulties. During follow-up, transabdoninal ultrasonography and cystoscopic examination identified no evidence of recurrence over the following 35 months.

\section{Discussion}

GISTs are mesenchymal tumors occurring in the gastrointestinal tract and their development is driven by mutant c-kit or platelet-derived growth factor receptor $\alpha$ genes. On mmunohistochemical examination, GISTs exhibit positive expression for CD117 or DOG-1. EGISTs are mesenchymal tumors occurring outside the gastrointestinal tract, with morphological, immunophenotypic and molecular biological characteristics very similar to those of GISTs (1). EGISTs are a rare clinical entity, accounting for $<5 \%$ of soft tissue tumors (2-4). Furthermore, EGISTs are even more rare in the urinary bladder. Following a literature review, we found 5 cases of patients with bladder 


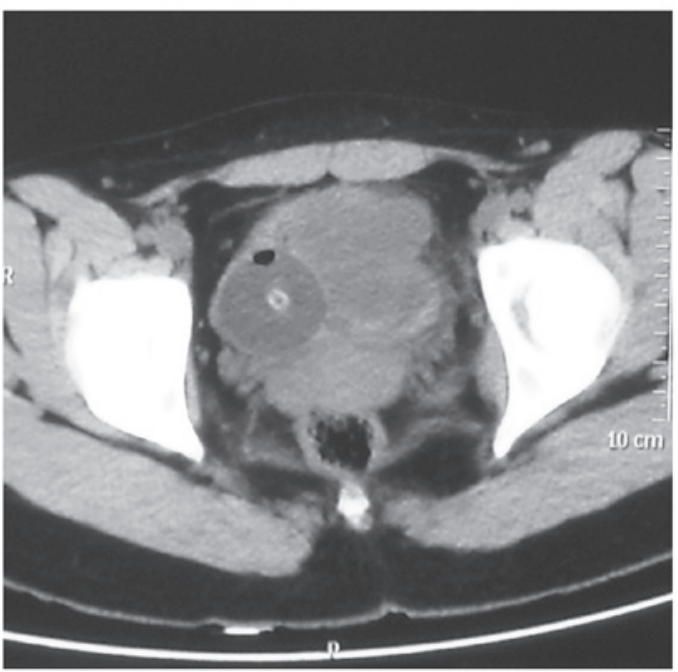

Figure 1. Computed tomography indicated an irregular soft tissue density mass, sized $5.7 \times 4.8 \mathrm{~cm}$, in the urinary bladder.
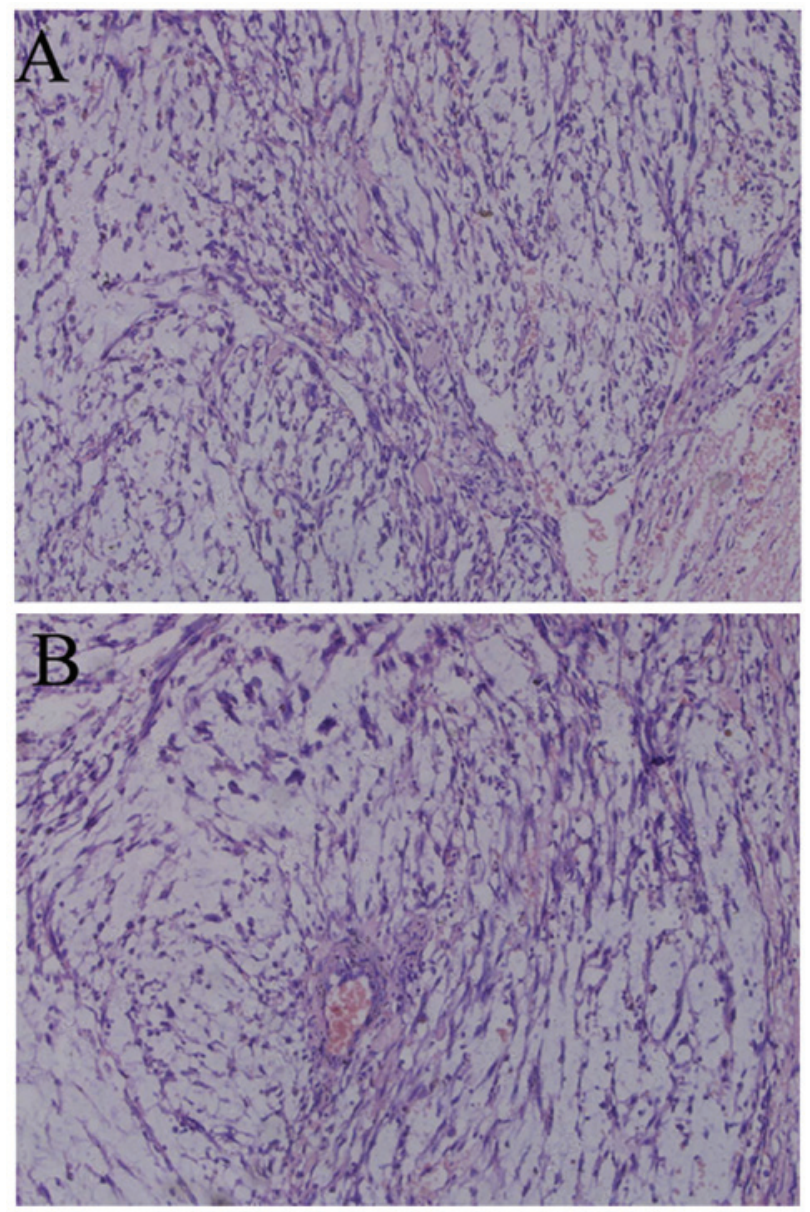

Figure 2. Hematoxylin and eosin-stained section of the tumor. (A) The tumor was composed of fusiform cells with abundant acidophilic cytoplasm and clavate nuclei, round at both ends and mostly blunt. (B) The cells were mostly arranged in interwoven bundles or swirls and were mildly atypical.

EGISTs, including 3 men and 2 women, aged 34-78 years (5-9). To the best of our knowledge, the patient in this study, aged 15 years, is the first non-adult patient presenting with EGIST, indicating that the occurrence of EGISTs is not limited to adults.

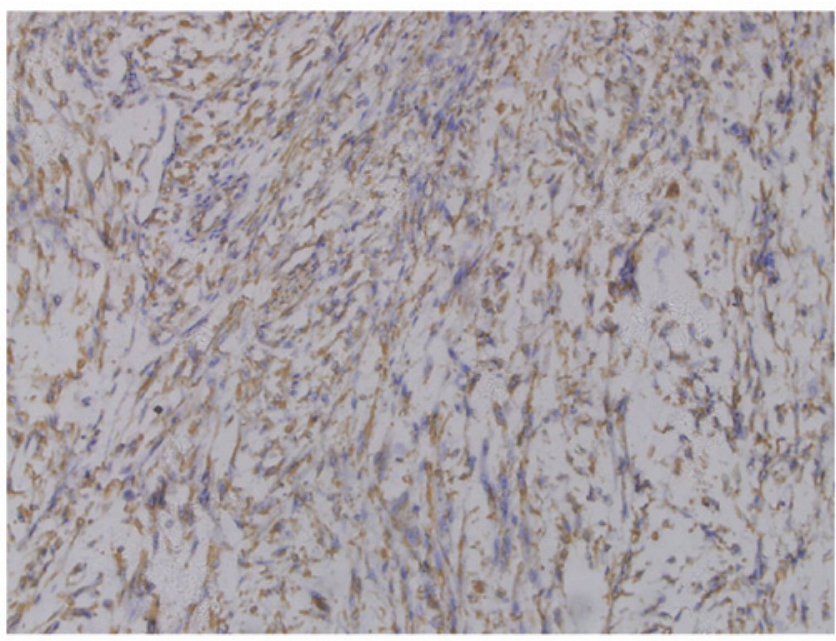

Figure 3. Tumor cells positively stained for CD117.

The clinical manifestations of EGISTs are non-specific. EGISTs of the bladder are characterized by larger tumor size and are mainly manifested by gross hematuria, which is often caused by bleeding due to tumor invasion of the mucous layer. EGISTs have no specific tumor markers. Furthermore, there are no specific diagnostic criteria for EGISTs on imaging examinations, such as B-mode ultrasound, CT, magnetic resonance imaging and position emission tomography/computed tomography. The current diagnostic criteria for EGISTs were developed according to the immunohistochemical diagnostic criteria for GISTs, i.e., mesenchymal tumors occurring outside the gastrointestinal tract, histologically consisting of fusiform, epithelioid or, occasionally, pleomorphic cells, in a bundle-like or diffuse arrangement; on immunohistochemical examination, the tumor cells are usually positive for CD117 or DOG-1 expression. The main clinical manifestation in our patient was hematuria and the lesion, which was located in the upper wall of the urinary bladder, was relatively large-sized and exhibited typical GIST histological morphology. The immunohistochemical examination revealed positive CD117 expression. Therefore, the diagnosis of EGIST was confirmed.

EGISTs are rare and their pathogenesis has not been fully elucidated. In this study, prompted by the GIST origin from pacemaker cells, also referred to as the interstitial cells of Cajal (ICC), which surround the gastrointestinal nerve plexus, we identified ICC cells in the gastrointestinal tract, as well as several other abdominal organs, extensively involving the bladder. Thus, we hypothesized that ICC carcinogenesis in the bladder is a primary cause of this disease. ICCs express the $\mathrm{c}$-kit protein. The c-kit proto-oncogene is located on human chromosome 4q11-12, of which the product is the growth factor receptor tyrosine kinase type III, a member of the immunoglobulin superfamily, also known as CD117. It was previously demonstrated that c-kit is expressed at relatively high levels in GISTs (up to 81-100\%) and is more sensitive compared to CD34 (10-12). However, in GISTs harbouring c-kit gene mutations, the positive rate of DOG-1 is $92 \%$ and that of CD117 is $81 \%$, making DOG-1 comparatively a more sensitive and specific indicator. Therefore, in cases of tumors morphologically suspicious for EGIST and negative for CD117 expression, DOG-1 is recommended for diagnosis $(13,14)$. 
The prognosis of EGIST appears to be related to morphological characteristics. Generally, compared to GISTs, a tumor may be determined as having malignant potential if it exhibits one of the following characteristics: i) Significantly atypical tumor cells, tumor necrosis, muscular layer invasion, ancient coin-like growth around the vessels and mitotic count $\geq 10 / 50 \mathrm{HPFs}$; or ii) mucosal invasion, perineural invasion, fatty infiltration, vascular invasion and lymph node metastasis. The more common the abovementioned findings, the higher the degree of malignancy $(15,16)$. However, these findings are derived from scattered onset locations and rare cases; therefore, the association between morphological characteristics and biological behavior requires further documentation for substantiation, particularly evidence-based medicine.

EGISTs are considered to be refractory to conventional radiotherapy and chemotherapy and resection is currently the preferred treatment option. The thoroughness of surgical treatment is closely associated with cure; therefore, a complete excision of the tumor by properly expanding the surgical scope is the key to EGIST treatment (17). By administering molecular-targeted drugs, such as imatinib, as neoadjuvant and adjuvant therapy for GISTs, small-scale clinical studies have achieved some clinical efficacy (18). However, in EGISTs, imatinib has been used in fewer cases; thus, our clinical experience is limited. The majority of these drugs are used for the prevention of EGIST relapse following surgery. The patient in our study did not receive any molecular-targeted drugs due to financial difficulties and there was no evidence of recurrence during follow-up over the next 35 months. The efficacy of molecular-targeted drugs, such as imatinib, in the treatment of EGISTs requires further support by clinical cases in the future.

\section{References}

1. Llenas-Garcia J, Guerra-Vales JM, Moreno A, Ibarrola C, Castelbon FJ, Fernandez-Ruiz M, Meneu JC, Ballestin C and Moreno E: Primary extragastrointestinal stromal tumors in the omentum and mesentery: a clinicopathological and immunohistochemical study. Hepatogastroenterology 55: 1002-1005, 2008.

2. Miettinen M, Monihan JM, Sarlomo-Rikala M, Kovatich AJ, Carr NJ, Emory TS and Sobin LH: Gastrointestinal stromal tumors/smooth muscle tumors (GISTs) primary in the omentum and mesentery: clinicopathologic and immunohistochemical study of 26 cases. Am J Surg Pathol 23: 1109-1118, 1999.

3. Reith JD, Goldblum JR, Lyles RH and Weiss SW: Extragastrointestinal (soft tissue) stromal tumors: an analysis of 48 cases with emphasis on histologic predictors of outcome. Mod Pathol 13: 577-585, 2000
4. Fletcher CD, Berman JJ, Corless C, et al: Diagnosis of gastrointestinal stromal tumors: A consensus approach. Hum Pathol 33: 459-465, 2002.

5. Lasota J, Carlson JA and Miettinen M: Spindle cell tumor of urinary bladder serosa with phenotypic and genotypic features of gastrointestinal stromal tumor. Arch Pathol Lab Med 124: 894-897, 2000.

6. Krokowski M, Jocham D, Choi H, Feller AC and Horny HP Malignant extragastrointestinal stromal tumor of bladder. J Urol 169: 1790-1791, 2003.

7. Mekni A, Chelly I, Azzouz H, Ben Ghorbel I, Bellil S, Haouet S, Kchir N, Zitouna M and Bellil K: Extragastrointestinal stromal tumor of the urinary wall bladder: case report and review of the literature. Pathologica 100: 173-175, 2008

8. Garcia-Segui A and Gascon M: Gastrointestinal stromal tumour in the urinary bladder. Actas Urol Esp 35: 124-125, 2011 (In Spanish).

9. Shin HS, Cho $\mathrm{CH}$ and Kum YS: Extragastrointestinal stromal tumor of the urinary bladder: a case report. Urol J 8: 165-167, 2011.

10. Kindblom LG, Remotti HE, Aldenborg F and Meis-Kindblom JM: Gastrointestinal pacemaker cell tumor (GIPACT): gastrointestinal stromal tumors show phenotypic characteristics of the interstitial cells of Cajal. Am J Pathol 152: 1259-1269, 1998

11. Hirota S, Isozaki K, Moriyama Y, et al: Gain-of-function mutations of c-kit in human gastrointestinal stromal tumors. Science 279: 577-580, 1998.

12. Sircar K, Hewlett BR, Huizinga JD, Chorneyko K, Berezin I and Riddell RH: Interstitial cells of Cajal as precursors of gastrointestinal stromal tumors. Am J Surg Pathol 23: 377-389, 1999.

13. Espinosa I, Lee CH, Kim MK, et al: A novel monoclonal antibody against DOG1 is a sensitive and specific marker for gastrointestinal stromal tumors. Am J Surg Pathol 32: 210-218, 2008.

14. Long KB, Butrynski JE, Blank SD, Ebrahim KS, Dressel DM, Heinrich MC, Corless CL and Hornick JL: Primary extragastrointestinal stromal tumor of the pleura: report of a unique case with genetic confirmation. Am J Surg Pathol 34: 907-912, 2010.

15. Miettinen $\mathrm{M}$ and Lasota J: Gastrointestinal stromal tumors: pathology and prognosis at different sites. Semin Diagn Pathol 23: 70-83, 2006.

16. Joensuu H: Risk stratification of patients diagnosed with gastrointestinal stromal tumor. Hum Pathol 39: 1411-1419, 2008.

17. Badalamenti G, Rodolico V, Fulfaro F, et al: Gastrointestinal stromal tumors (GISTs): focus on histopathological diagnosis and biomolecular features. Ann Oncol 18 (Suppl 6): vi136-vi140, 2007.

18. Dematteo RP, Ballman KV, Antonescu CR, et al; American College of Surgeons Oncology Group (ACOSOG) Intergroup Adjuvant GIST Study Team: Adjuvant imatinib mesylate after resection of localised, primary gastrointestinal stromal tumour: a randomised, double-blind, placebo-controlled trial. Lancet 373: 1097-1104, 2009. 